emphasise the relationship between HIV and other STIs and the importance of having health workers counsel their STD patients and their sexual partners on the need for an HIV test. MU-JHU supported districts health workers by training them.

Results Between July 2010 and Dec 2011 over 350 health workers in 19 central districts were trained in syndromic management of STDs with an emphasis an counselling patients and referring them for HIV testing. Over 17,613 clients with STDs have since undergone HIV testing in those facilities; over 18,000 clients with STDs have been referred for HIV testing to outside facilities that did not have testing services. HIV rates in STD patients overage 18\%.

Conclution Operational level health workers handling STD patients need basic counselling training to discuss HIV/AIDS with their clients. Targeting STD patients for routine HIV counselling and testing this is essential to reach more people infected with HIV.

\section{P4.106 NIGHT HARM REDUCTION SERVICES ACCESSIBILITY FOR PREVENTION: A MODEL OF SUCCESS}

doi:10.1136/sextrans-2013-051184.1003

\section{O D Obi Peter. Traffina Foundation for Community Health, Asokoro, Nigeria}

Background There are lots of interventions among men who have sex with men Cross River, new evidence suggests that many recent HIV infections are connected with the use of Meth-am-phet-a-mine (C10H15N) among bisexual HIV testers in the state last year (IBBSS 2007). HIV incidence among MA users was 3.3\% compared to $1.1 \%$ among non-MA users.

Methods The purpose of the night harm reduction services was to ascertain the feasibility and uptake of harm reduction services by a late night population of MSM. The night services included: needle exchange, harm reduction information, oral HIV testing, and urine based sexually transmitted infection (STI) testing accompanied by counselling and consent procedures. The study had two components: harm reduction outreach and a behavioural survey. For 2 months in the year 2008, we provided mobile base harm reduction services in three neighbourhoods in Calabar - Cross River State from evening till midnight fall.

Results We exchanged 1090 needles in 121 needle exchange visits, distributed 3200 condoms for both male and provided 18 HIV tests and 8 opportunistic infections tests. About 45 MSM and lesbian friends 25 of them enrolled in the study conducted. The study population of MSM was characterised by low levels of income, stigma and discrimination. The level of education whose ages ranged from $18-55$. Fifty percent used MA in the last 3 months before; almost $15 \%$ used MA every day in the same time frame contracting deadly infections.

Conclusion It is fair to believe night work services can only be acceptable to our MSMs whose lives the government has put in danger over their sexual rights and orientation. Harm reduction: the next generation is possible only if there is a conducive atmosphere for them and services provided affordably and comprehensively.

\section{P4.107 PARENTS CHILD COMMUNICATION IMPROVEMENT AMONG HIV INFECTED \& AFFECTED CHILDREN}

doi:10.1136/sextrans-2013-051184.1004

S Das(Konar), N Panda, M Kundu, R Das, S Chowdhury, R Sonkar, R Basu, A RoyChowdhury. Child in Need Institute, Kolkata, India

One of the major impacts of the HIV \& AIDS epidemic on global society is seen in the increase number of children being infected \& affected by AIDS. There is a need for one programme to addressing these issues to support of those children $\&$ their parents who are suffering from AIDS. CINI was working on 300 children \& their parents of IICCHAA project since 2006. The memory work sessions taught the parents and children to accept reality and cope to live in their own surrounding by using their creative minds to open up opportunities of self-discovery and self- sustaining. In these sessions, children and parents started to share by transparent communication with families, providing care and support, unified planning for future by taking everybody's opinion and views according to their needs.

CINI assessed this programme in two levels -Developing the resilience of the Children andEmpowerment of the children. 300 children \& their parents/family members were empowered through memory work sessions.

A comparative analysis of the current situation shows that:

- The ratio of Communication skill has been increased from $17.3 \%$ to $80.5 \%$

- The leadership qualities boosted from $12 \%$ to $72 \%$

- The Children developed themselves for taking care of parents, earlier the ratio was $9 \%$ which is raises up to $57 \%$

- Parents are becoming empowered to disclose their status. Presently the percentage increases from 15 to 90

- Earlier the Parents were not able to prepare any succession plan for safe guard of their children. At present it is developed up to $97 \%$

The PLHIVs can improve the quality of their lives, if IICCHAA will include in the national programme.

\section{P4.108 WITH WHOM DID YOU HAVE SEX? EVALUATION OF A PARTNER NOTIFICATION TRAINING FOR STI PROFESSIONALS USING MOTIVATIONAL INTERVIEWING}

doi:10.1136/sextrans-2013-051184.1005

${ }^{1}$ E Op de Coul, ${ }^{2} \mathrm{R}$ Spijker, ${ }^{1} \mathrm{~F}$ van Aar, ${ }^{1} \mathrm{Y}$ van Weert, ${ }^{3} \mathrm{M}$ de Bruin. ${ }^{1} R / V M$, Bilthoven, The Netherlands; ${ }^{2}$ STI Aids The Netherlands, Amsterdam, The Netherlands; ${ }^{3}$ Amsterdam School of Communications Research, University of Amsterdam, Amsterdam, The Netherlands

Objective To enhance partner notification (PN) practises in STI clinics a newly developed $\mathrm{PN}$ training based on motivational interviewing (MI) was offered to STI professionals and evaluated.

Methods The effectiveness of PN training on the professionals' attitude, self-efficacy, and behaviour toward PN was examined with a within-subject and between-subject comparison. Just before and three months after delivery of the training, a questionnaire with 7 -point Likert scales was completed by the intervention group $(n=54)$. To rule out that any effects could be caused by population trends or questionnaire completion, a non-randomly selected sample of controls $(n=37)$ also completed the questionnaires. Questionnaire items were examined separately and grouped into constructs of attitude, self-efficacy and behaviour

Results In the within-subject (pre-post) comparison, positive changes were observed in 9/30 of the items, as well as in the overall constructs Self-efficacy, Skills: Implementation PN protocol, and Skills: coping with client resistance. In the control group no significant changes were observed. However, comparing the change scores between the intervention and control group, 5 items and the construct Self-efficacy were no longer significant.

Conclusions Despite that the PN training for STI professionals had no effect on attitudes or self-efficacy, significant improvements were observed in the most important outcome variables, namely those on skills. In studies were cluster randomised controlled studies are not feasible, selecting a convenient control sample seems to offer a more rigorous test of hypotheses than pre-post evaluations, presumably by controlling for population trends and mere measurement effects.

\section{P4.109 IMPACT OF PEER EDUCATORS TRAINING EX FIGHTERS BURUNDI AS A STRATEGY TO REDUCE RISK BEHAVIOURS FAVORING THE TRANSMISSION OF HIV/AIDS}

doi:10.1136/sextrans-2013-051184.1006

F Ndayimirije. forum des anciens combattants pour la lutte contre le VIH/SIDa l'amélioration de la santé et de, Bujumbura, Burundi 